\title{
Women with fibromyalgia struggled to gain integrity, understanding, symptom relief, and freedom in their everyday lives
}

Söderberg S, Lundman B, Norberg A. Struggling for dignity: the meaning of women's experiences of living with

fibromyalgia. Qual Health Res 1999 Sep;9:575-87.

QUESTION: For women, what is the meaning of living with fibromyalgia?

Design

A phenomenological hermeneutic study.

\section{Setting}

An outpatient rheumatological clinic in a large hospital in northern Sweden.

\section{Participants}

14 women aged 35-50 years (mean age 43 y) with fibromyalgia (American College of Rheumatology criteria) who had been treated at the clinic were recruited by purposive sampling. The women had experienced symptoms for $1-25$ years and had been diagnosed for $0.5-6$ years.

\section{Methods}

Before clinical treatment, individual narrative interviews of 15-60 minutes were done. Women were asked to talk about symptoms, daily life, relationships with others, and thoughts and feelings about fibromyalgia. Audiotaped interviews were transcribed verbatim, reviewed for accuracy, and analysed using naïve understanding and structural analysis. This process began with naïve reading, aimed at acquiring a sense of the whole and was followed by structural analysis, aimed at identifying patterns of meaningful consistency and seeking explanations. Analysis was completed with interpretation of the whole text, in which the previous 2 steps, together with the authors' pre-understanding, were combined into a new comprehensive understanding.

\section{Main findings}

3 interrelated themes were identified. The first theme, loss of freedom, emerged from feelings of a body in pain and other bodily sensations, feelings of fatigue and loss of energy, living a changed everyday life in order to adapt to the illness, and economic restrictions and strain. The second theme, threat to integrity, referred to a loss of credibility and the invisibility of the illness. Women felt they were not believed when seeking help for their symptoms because the illness was not visible. They also felt a lack of personal knowledge about fibromyalgia. They perceived a lack of understanding and a negative attitude from healthcare professionals, relatives, friends, and workmates. The third theme, a struggle to achieve understanding and relief, described the women's search for explanations relating to the diagnosis of fibromyalgia. Women also sought relief from different treatments and struggled to plan their daily lives in accordance with their illness.

\section{Conclusion}

Women living with fibromyalgia experienced a loss of freedom in their everyday lives because of pain, fatigue, and economic struggles; felt that their integrity was threatened because of the invisible nature of their illness and society's lack of understanding of it; and struggled to understand their illness and to gain relief.

\section{COMMENTARY}

Fibromyalgia is a chronic illness that is most common among middle aged women and characterised by chronic diffuse musculoskeletal pain, tender points, and fatigue. The findings of this study by Söderberg et al concur with the results of the few existing qualitative investigations. The concept of struggle seems to be a common feature for women with fibromyalgia. This analysis explores the "struggle for dignity", whereas Schaefer ${ }^{1}$ described the "struggle to maintain balance". A study by Gaston-Johansson showed that women with fibromyalgia had a more negative self image, reported more pain, and experienced more limitations than did women with rheumatoid arthritis. ${ }^{2}$

It seems proper to use the findings of this well designed study as one basis for practice with patients with fibromyalgia. An obvious strength of the study is the description of the struggles and problems of fibromyalgia in the words of the women affected. This study included a relatively small number of women, all from 1 country; concerns expressed by women in other countries could be different.

Being diagnosed with a "vague" disease, one that has an unknown aetiology, can cause women to feel that their symptoms are psychosomatic. These beliefs may be reinforced by health professionals, families, and friends. As with other diseases that occur more frequently in women, patients may be made to feel as though they are malingering, which can produce feelings of guilt, helplessness, and loss of control.

Nursing practice should be based within a context of acceptance of the client and her feelings. Accepting and supporting patients with fibromyalgia provides validation of the patient's perceptions and her value as a human being. The nurse may serve as advocate in representing women to those who discount these symptoms. As educators, nurses can assist women to discover information about the disease and measures to alleviate symptoms. Finally, nurses can encourage women to see other helping professionals or to become involved in fibromyalgia support groups.

M Kay Libbus, RN, DrPH Associate Professor, Sinclair School of Nursing University of Missouri-Columbia Columbia, Missouri, USA

1 Schaefer KM. Struggling to maintain balance: a study of women living with fibromyalgia. J Adv Nurs 1995;21:95-102.

2 Gaston-Johansson F, Gustafsson M, Felldin R, et al. A comparative study of feelings, attitudes and behaviors of patients with fibromyalgia and rheumatoid arthritis. Soc Sci Med 1990;31:941-7. 